\title{
Thermal dispersion effects on convective heat and mass transfer in an Ostwald de Waele nanofluid flow in porous media
}

\author{
Peri K Kameswaran and Precious Sibanda*
}

${ }^{\text {"Correspondence: }}$

sibandap@ukzn.ac.za

School of Mathematics, Statistics

and Computer Science, University

of KwaZulu-Natal, Private Bag X01

Scottsville, Pietermaritzburg, 3209,

South Africa

\section{Springer}

\begin{abstract}
In this paper we investigate the effects of thermal dispersion on non-Newtonian power-law nanofluid flow over an impermeable vertical plate. We use a mathematical model for the nanofluid that incorporates the effects of nanoparticle Brownian motion and thermophoresis. The non-Newtonian nature of the fluid is modeled by the power-law index $n$, and both cases of shear thinning and thickening are investigated. The governing set of equations is solved numerically using the shooting technique. The effects of physical and fluid parameters on the properties are determined for both instances of aiding and opposing buoyancy force. We show, inter alia, that for fixed thermal dispersion coefficient values, the fluid temperature within the boundary layer decreases as the power-law index increases for both aiding and opposing buoyancy case.
\end{abstract}

Keywords: non-Newtonian nanofluid; natural convection; thermal dispersion; porous medium

\section{Introduction}

The study of a non-Newtonian power-law fluid model is important in simulating the rheological characteristics of numerous fluids such as used in food preparation, bio-chemical and pharmaceutical industries. Many interesting applications of non-Newtonian powerlaw fluids were presented by Shenoy [1]. Such non-Newtonian fluids may be important in, for instance, the physiological transport of biological solutions, polymer melts, paints, etc. (Ellahi et al. [2]). Rastogi and Poulikakos [3] examined the problem of double diffusion from a plate in a porous medium saturated with a non-Newtonian power-law fluid. The effect of double dispersion on natural convection heat and mass transfer in a nonNewtonian fluid saturated non-Darcy porous medium was studied by Kairi et al. [4]. They observed that the heat transfer rate increases with the thermal dispersion effect for both pseudoplastics and dilatant fluids.

In recent years, the study of convective heat transfer in nanofluids has received a lot of attention. Nanofluids which are suspensions of solid nanoparticles in conventional liquids like water, oil and ethylene glycol have many potential applications in industry. Convection in nanofluid flows in porous media has also received attention due to its potential application in materials processing and solar energy collector devices. Nield and Kuznetsov [5] extended the Cheng-Minkowycz problem for heat transfer in porous media to nanofluids. Uddin et al. [6] used the lie group analysis to study non-Newtonian nanofluid flow in a

○2013 Kameswaran and Sibanda; licensee Springer. This is an Open Access article distributed under the terms of the Creative Commons Attribution License (http://creativecommons.org/licenses/by/2.0), which permits unrestricted use, distribution, and reproduction in any medium, provided the original work is properly cited. 
porous medium with internal heat generation. They investigated heat transfer rates and showed that the rates decrease with increasing power-law index, particle Brownian motion, thermophoresis and fluid buoyancy. The boundary layer formed by a non-Newtonian flow over a vertical plate in a porous medium saturated with a nanofluid was studied by Hady et al. [7]. In their study, they found that the reduced Nusselt number increases with the power-law index and the Lewis parameter. Gorla and Khan [8] studied natural convection in boundary layer flow over a vertical cylinder embedded in a porous medium saturated with a nanofluid. Free convection in boundary layer flow over horizontal and vertical plates was investigated by, among others, Gorla and Chamkha $[9,10]$.

In this article we investigate the effects of thermal dispersion on a non-Newtonian power-law nanofluid over an impermeable vertical plate. The effects of nanoparticle Brownian motion and thermophoresis on the heat and mass transfer characteristics are analyzed. Numerical solutions are obtained using the shooting method.

\section{Mathematical analysis}

We consider the steady two-dimensional boundary layer of a non-Newtonian nanofluid over a vertical impermeable wall embedded in a porous medium. The $x$-coordinate is taken along the plate and $y$-coordinate is normal to it. Temperature $T$ and nanoparticle fraction $C$ are taken as constant values $T_{w}$ and $C_{w}$, respectively. The ambient values of $T$ and $C$ are denoted by $T_{\infty}$ and $C_{\infty}$. The Oberbeck-Boussinesq approximation was employed and homogeneity and local thermal equilibrium in the porous medium was assumed. Using the standard boundary layer approximations, we can write the governing equations as follows:

$$
\begin{aligned}
& \frac{\partial u}{\partial x}+\frac{\partial v}{\partial y}=0, \\
& n u^{n-1} \frac{\partial u}{\partial y}=\frac{\left(1-C_{\infty}\right) K g \beta \rho f_{\infty}}{\mu} \frac{\partial T}{\partial y}-\frac{\left(\rho_{p}-\rho f_{\infty}\right) K g}{\mu} \frac{\partial C}{\partial y}, \\
& u \frac{\partial T}{\partial x}+v \frac{\partial T}{\partial y}=\frac{\partial}{\partial y}\left(\alpha_{e} \frac{\partial T}{\partial y}\right)+\tau\left\{D_{B} \frac{\partial T}{\partial y} \frac{\partial C}{\partial y}+\frac{D_{T}}{T_{\infty}}\left(\frac{\partial T}{\partial y}\right)^{2}\right\}, \\
& \frac{1}{\varepsilon}\left(u \frac{\partial C}{\partial x}+v \frac{\partial C}{\partial y}\right)=D_{B} \frac{\partial^{2} C}{\partial y^{2}}+\left(\frac{D_{T}}{T_{\infty}}\right) \frac{\partial^{2} T}{\partial y^{2}},
\end{aligned}
$$

where

$$
\alpha_{e}=\alpha_{m}+\gamma d u, \quad \alpha_{m}=\frac{k}{(\rho c)_{f}} \quad \text { and } \quad \tau=\frac{\varepsilon(\rho c)_{p}}{(\rho c)_{f}} .
$$

The boundary conditions for equations (1)-(4) are given in the form:

$$
\begin{aligned}
& \nu=0, \quad T=T_{w}, \quad C=C_{w}, \quad \text { at } y=0, \\
& u \rightarrow 0, \quad T \rightarrow T_{\infty}, \quad C \rightarrow C_{\infty}, \quad \text { as } y \rightarrow \infty .
\end{aligned}
$$

Here $u, v$ are velocity components in vertical $(x)$ and horizontal $(y)$ directions, $n$ is the power law index, $K$ is the modified permeability of the porous medium, $g$ is the gravitational acceleration, $\beta$ is the volumetric expansion of the base fluid, $\rho_{f}$ is the density of the base fluid, $\mu$ is the consistency index of the power law fluid, $T$ is the local temperature, 
$C$ is the nanoparticle volume fraction, $\rho_{p}$ is the density of nanoparticle, $\alpha_{e}$ is the effective thermal diffusivity of the porous medium that can be written as $\alpha_{e}=\alpha_{m}+\gamma d u, \alpha_{m}$ is the molecular thermal diffusivity, $\gamma d u$ represent the thermal diffusivity, $\gamma$ is the mechanical thermal dispersion coefficient. This value lies between $1 / 7$ and 1/3 (see Kairi et al. [4]), $d$ is the pore diameter, $\tau$ is ratio of the effective heat capacity of the nanoparticle material and the heat capacity of the fluid, $D_{B}$ is the Brownian motion coefficient, $D_{T}$ is the thermophoretic diffusion coefficient, $\varepsilon$ is the porosity of the porous medium, respectively, $k$ is the effective thermal conductivity of the porous medium, $(\rho c)_{f}$ is the heat capacity of the nanofluid and $(\rho c)_{p}$ is the effective heat capacity of the nanoparticle material.

The modified permeability of the porous medium $K$ of the non-Newtonian power law fluid is defined as

$$
K=\frac{1}{2 c_{t}}\left(\frac{n \varepsilon}{3 n+1}\right)^{n}\left(\frac{50 k^{*}}{3 \varepsilon}\right)^{\frac{n+1}{2}}, \quad k^{*}=\frac{\varepsilon^{3} d^{2}}{150(1-\varepsilon)^{2}}
$$

and

$$
c_{t}= \begin{cases}\frac{25}{12} & {[11]} \\ \frac{2}{3}\left(\frac{8 n}{9 n+3}\right)^{n}\left(\frac{10 n-3}{6 n+1}\right)\left(\frac{75}{16}\right)^{3(10 n-3) /(10 n+11)} & {[12]}\end{cases}
$$

For $n=1, c_{t}=25 / 12$. Equation (1) is satisfied by introducing a stream function $\psi(x, y)$ such that

$$
u=\frac{\partial \psi}{\partial y} \quad \text { and } \quad v=-\frac{\partial \psi}{\partial x}
$$

where $\psi=\alpha_{m} \operatorname{Ra}_{x}^{\frac{1}{2}} f(\eta), f(\eta)$ is the dimensionless stream function, $\eta=(y / x) \operatorname{Ra}_{x}^{\frac{1}{2}}$ and $\operatorname{Ra}_{x}$ is the local Rayleigh number given by

$$
\operatorname{Ra}_{x}=\frac{x}{\alpha_{m}}\left(\frac{\left(1-C_{\infty}\right) K g \beta \rho f_{\infty} \Delta T}{\mu}\right)^{\frac{1}{n}} .
$$

The velocity components are given by

$$
u=\left(\frac{\alpha_{m}}{x}\right) \operatorname{Ra}_{x} f^{\prime}(\eta) \quad \text { and } \quad v=\left(\frac{\alpha_{m}}{2 x}\right) \operatorname{Ra}_{x}^{\frac{1}{2}}\left[\frac{y}{x} \operatorname{Ra}_{x}^{\frac{1}{2}} f^{\prime}(\eta)-f(\eta)\right] .
$$

The temperature and concentration are presented as

$$
T=T_{\infty}+\Delta T \theta(\eta) \quad \text { and } \quad C=C_{\infty}+\Delta C \phi(\eta)
$$

where $\theta(\eta)$ and $\phi(\eta)$ are the dimensionless temperature and dimensionless concentration. On using equations (8) and (9), equations (2), (3), (4) and (6) transform into the following two-point boundary value problem:

$$
\begin{aligned}
& n\left(f^{\prime}\right)^{n-1} f^{\prime \prime}-\theta^{\prime}+\mathrm{Nr} \phi^{\prime}=0, \\
& \theta^{\prime \prime}+\frac{1}{2} f \theta^{\prime}+\gamma \operatorname{Ra}_{d}\left(f^{\prime} \theta^{\prime \prime}+f^{\prime \prime} \theta^{\prime}\right)+\mathrm{Nb} \theta^{\prime} \phi^{\prime}+\mathrm{Nt} \theta^{2}=0,
\end{aligned}
$$




$$
\phi^{\prime \prime}+\frac{1}{2} \operatorname{Lef} \phi^{\prime}+\frac{\mathrm{Nt}}{\mathrm{Nb}} \theta^{\prime \prime}=0
$$

subject to the boundary conditions

$$
\begin{aligned}
f(0) & =0, & f^{\prime}(\infty) & \rightarrow 0, \\
\theta(0) & =1, & \theta(\infty) & \rightarrow 0, \\
\phi(0) & =1, & \phi(\infty) & \rightarrow 0,
\end{aligned}
$$

where the primes denote differentiation with respect to $\eta$. The non-dimensional constants in equations (10)-(12) are the buoyancy ratio parameter $\mathrm{Nr}$, the coefficient of thermal dispersion $\gamma$, the pore diameter dependent Rayleigh number $\mathrm{Ra}_{d}$, the Brownian motion parameter $\mathrm{Nb}$, the thermophoresis parameter $\mathrm{Nt}$ and the Lewis number Le. These are defined as

$$
\begin{aligned}
& \mathrm{Nr}=\frac{\left(\rho_{p}-\rho f_{\infty}\right) \Delta C}{\left(1-C_{\infty}\right) \beta \rho f_{\infty} \Delta T}, \quad \operatorname{Ra}_{d}=\frac{d}{\alpha_{m}}\left(\frac{\left(1-C_{\infty}\right) K g \beta \rho f_{\infty} \Delta T}{\mu}\right)^{\frac{1}{n}}, \\
& \mathrm{Nb}=\frac{\tau D_{B} \Delta C}{\alpha_{m}}, \quad \mathrm{Nt}=\frac{\tau D_{T} \Delta T}{T_{\infty} \alpha_{m}}, \quad \text { Le }=\frac{\alpha_{m}}{\varepsilon D_{B}} .
\end{aligned}
$$

\section{Heat and mass transfer coefficients}

The local heat flux at the vertical wall is given by

$$
q_{w}=-k\left[\frac{\partial T}{\partial y}\right]_{y=0} .
$$

The local Nusselt number is defined as

$$
\mathrm{Nu}_{x}=\frac{x q_{w}}{k\left(T_{w}-T_{\infty}\right)}
$$

where $k$ is the effective thermal conductivity of the porous medium, which is the sum of the molecular thermal conductivity $k_{m}$ and the dispersion thermal conductivity $k_{d}$. Using equation (17) in equation (18), the dimensionless Nusselt number can be represented as

$$
\mathrm{Nu}_{x}=-\mathrm{Ra}_{x}^{1 / 2}\left[1+\gamma \operatorname{Ra}_{d} f^{\prime}(0)\right] \theta^{\prime}(0) .
$$

The mass flux at the vertical wall is given by

$$
q_{m}=-D\left[\frac{\partial C}{\partial y}\right]_{y=0}=-D\left(C_{w}-C_{\infty}\right) \frac{1}{x} \operatorname{Ra}_{x}^{\frac{1}{2}} \phi^{\prime}(0) .
$$

The local Sherwood is defined as

$$
\mathrm{Sh}_{x}=\frac{x q_{m}}{D\left(C_{w}-C_{\infty}\right)} .
$$

Using (20) in (21) the dimensionless Sherwood number is obtained as

$$
\mathrm{Sh}_{x}=-\mathrm{Ra}_{x}^{1 / 2} \phi^{\prime}(0) .
$$




\section{Numerical solution}

The set of ordinary differential equations (10)-(12) along with the boundary conditions (13)-(15) form a two-point boundary value problem (BVP) that is solved using the shooting method. Coupled ordinary differential equations (10)-(12) which are second-order in $f, \theta$ and $\phi$ are reduced to a system of six simultaneous equations in six unknowns. In order to numerically solve this system of equations, we require six initial conditions, but one initial condition in each of $f, \theta$ and $\phi$ is known. However, the values of $f^{\prime}, \theta$ and $\phi$ are known at $\eta \rightarrow \infty$. By using these three conditions, we have to provide the unknown initial conditions at $\eta=0$ by using the shooting technique. The main step in this method is to choose an appropriate finite value of $\eta \rightarrow \infty$. The solution procedure is repeated with different values of $\eta \rightarrow \infty$ until two successive values of $f^{\prime}(0), \theta^{\prime}(0)$ and $\phi^{\prime}(0)$ differ only by a specified significant digit. The value of $\eta$ may change for a different set of physical parameters. Once the appropriate value of $\eta$ is determined, the coupled boundary value problem given by equations (10)-(12) is solved numerically using the Runge-Kutta integration scheme. Equations (10)-(12) can be written as

$$
\begin{aligned}
f^{\prime \prime} & =\frac{\theta^{\prime}-\mathrm{Nr} \phi^{\prime}}{n\left(f^{\prime}\right)^{n-1}}, \\
\theta^{\prime \prime} & =\frac{-0.5 f \theta^{\prime}-\gamma \operatorname{Ra}_{d} f^{\prime \prime} \theta^{\prime}-\mathrm{Nb} \theta^{\prime} \phi^{\prime}-\mathrm{Nt} \theta^{\prime 2}}{1+\gamma \operatorname{Ra}_{d} f^{\prime}}, \\
\phi^{\prime \prime} & =-0.5 \operatorname{Le} f \phi^{\prime}-\frac{\mathrm{Nt}}{\mathrm{Nb}} \theta^{\prime \prime} .
\end{aligned}
$$

We define new variables

$$
f_{1}=f, \quad f_{2}=f^{\prime}, \quad f_{3}=\theta, \quad f_{4}=\theta^{\prime}, \quad f_{5}=\phi, \quad f_{6}=\phi^{\prime},
$$

so that the three second-order coupled differential equations transform to six first-order differential equations

$$
\begin{aligned}
& f_{1}^{\prime}=f_{2}, \\
& f_{2}^{\prime}=\frac{f_{4}-\mathrm{Nr} f_{6}}{n\left(f_{2}\right)^{n-1}}, \\
& f_{3}^{\prime}=f_{4}, \\
& f_{4}^{\prime}=\frac{-0.5 f f_{4}-\gamma \mathrm{Ra}_{d} f_{2}^{\prime} f_{4}-\mathrm{Nb}_{4} f_{6}-\mathrm{Nt} f_{4}^{2}}{1+\gamma \mathrm{Ra}_{d} f_{2}}, \\
& f_{5}^{\prime}=f_{6}, \\
& f_{6}^{\prime}=-0.5 \text { Leff }-\frac{\mathrm{Nt}}{\mathrm{Nb}} f_{4}^{\prime},
\end{aligned}
$$

where the primes denote differentiation with respect to $\eta$. The boundary conditions are

$$
\begin{array}{ll}
f_{1}(0)=0, & f_{2}(\infty) \rightarrow 0, \\
f_{3}(0)=1, & f_{3}(\infty) \rightarrow 0, \\
f_{5}(0)=1, & f_{5}(\infty) \rightarrow 0 .
\end{array}
$$


Table 1 Comparison of $\mathrm{Nu}_{x} / \mathbf{R a}_{x}^{1 / 2}$ for different values of power law index $n$

\begin{tabular}{lll}
\hline Exponent $\boldsymbol{n}$ & $\mathbf{N u}_{\boldsymbol{x}} / \mathbf{R a}_{\boldsymbol{x}}^{\mathbf{1 / 2}}$ & \\
\cline { 2 - 3 } & $\mathbf{M o h a m m a d i e n ~ a n d ~ E l - A m i n ~ [ 1 3 ] ~}$ & Present \\
\hline 0.5 & - & 0.37675 \\
1 & 0.44390 & 0.44375 \\
1.5 & - & 0.47635 \\
\hline
\end{tabular}

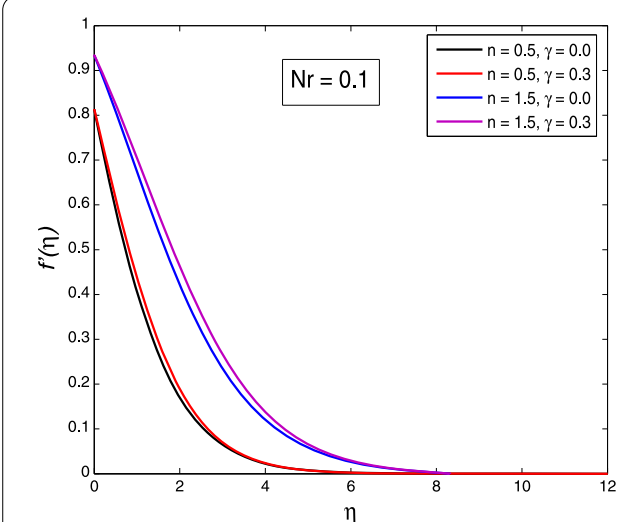

(a)

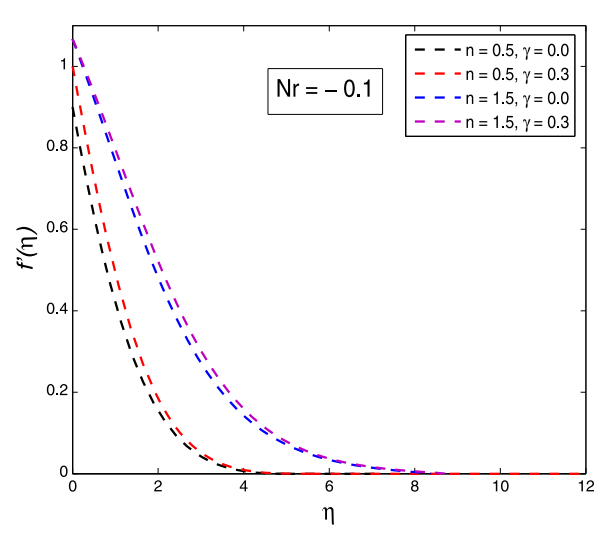

(b)

Figure 1 Variation of velocity profiles with similarity variable $\eta$ for (a) aiding buoyancy $(\mathrm{Nr}>0)$ and (b) opposing buoyancy $(\mathrm{Nr}<0)$, when $\operatorname{Ra}_{d}=1, \mathrm{Nb}=\mathrm{Nt}=0.3, \mathrm{Le}=1$.

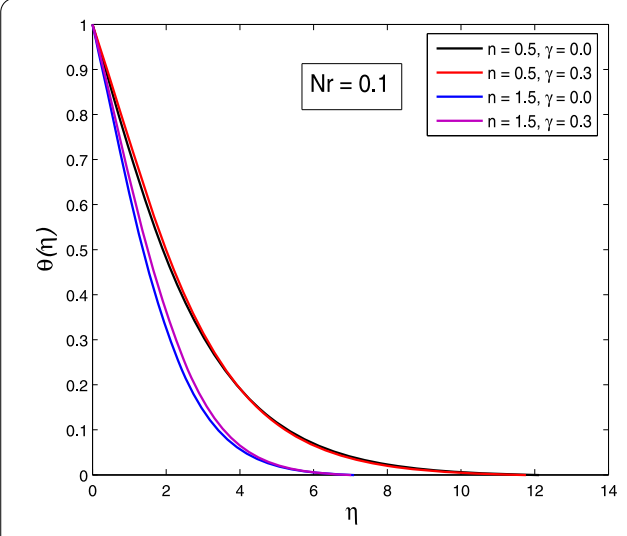

(a)

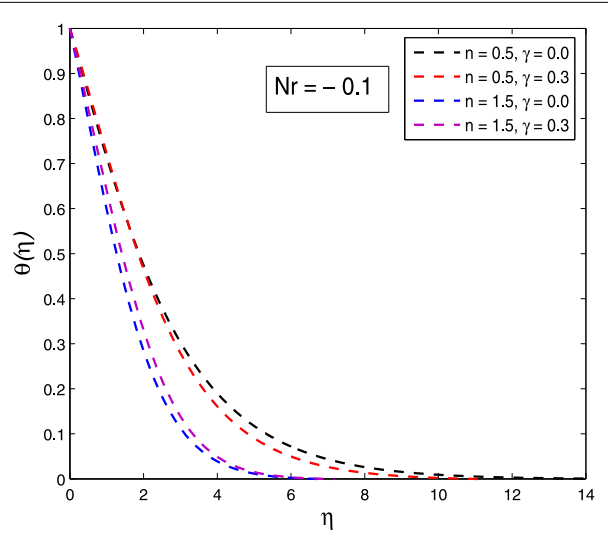

(b)

Figure 2 Variation of temperature profiles with similarity variable $\eta$ for (a) aiding buoyancy $(\mathrm{Nr}>0)$ and (b) opposing buoyancy $(\mathrm{Nr}<0)$, when $\mathrm{Ra}_{d}=1, \mathrm{Nb}=\mathrm{Nt}=0.3, \mathrm{Le}=1$.

\section{Results and discussion}

The results obtained here are accurate up to three decimal places and validation was done by comparison of the Nusselt number with the results obtained by Mohammadien and El-Amin [13] in the absence of radiation and non-Darcy parameters. It is observed from Table 1 that heat transfer rate increases with an increase in power-law index. The present results are in good agrement with [13] when $n=1, \mathrm{Nr}=0, \gamma=\mathrm{Ra}_{d}=\mathrm{Nb}=\mathrm{Nt}=0, \mathrm{Le}=0$. The effects of various physical and fluid parameters are shown in Figures 1-12. 


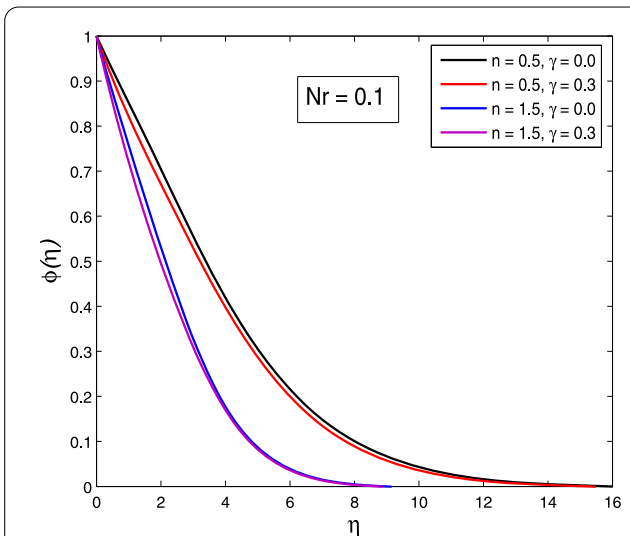

(a)

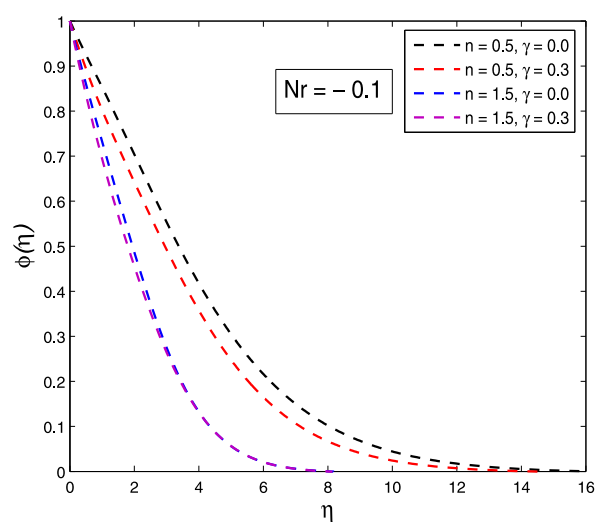

(b)

Figure 3 Variation of concentration profiles with similarity variable $\eta$ for (a) aiding buoyancy ( $\mathrm{Nr}>0$ ) and (b) opposing buoyancy $(\mathrm{Nr}<0)$, when $\mathrm{Ra}_{d}=1, \mathrm{Nb}=\mathrm{Nt}=0.3, \mathrm{Le}=1$.

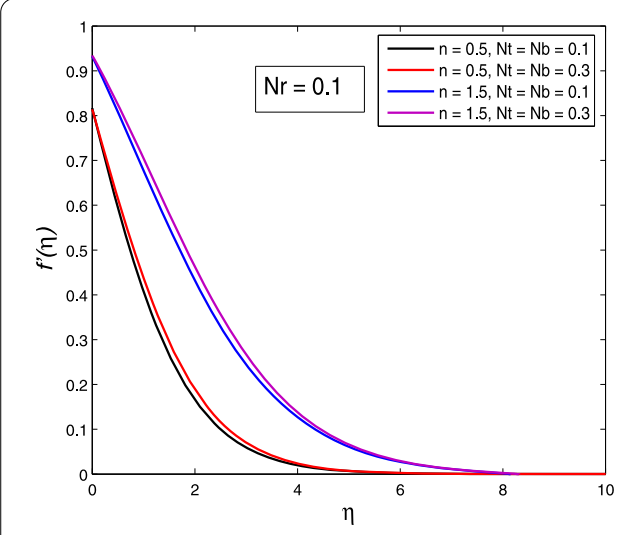

(a)

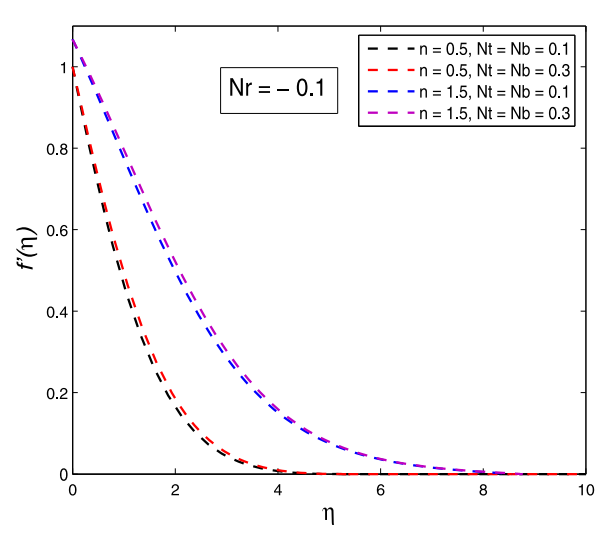

(b)

Figure 4 Variation of velocity profiles with similarity variable $\eta$ for (a) aiding buoyancy $(\mathrm{Nr}>0)$ and (b) opposing buoyancy $(\mathrm{Nr}<0)$, when $\gamma=0.3, \operatorname{Ra}_{d}=1$, Le $=1$.

Figures 1-3 illustrate the effects of $n$ and $\gamma$ on velocity, temperature and nanoparticle volume fraction profiles, respectively for both aiding and opposing buoyancy cases. Velocity profiles diminish with a decrease in the thermal dispersion coefficient for pseudoplastics and dilatants in the aiding buoyancy case. The same trend is observed in the case of opposing buoyancy, but for the case of a dilatant, the decrease in the boundary layer thickness is more significant compared to the case of aiding buoyancy.

The temperature profiles are shown in Figure 2. For a fixed coefficient of thermal dispersion, the thermal boundary layer thickness decreases with an increase in the power-law index for both aiding and opposing buoyancy. These results are qualitatively similar to those obtained by Kairi et al. [4] in the case of a clear fluid. Thermal dispersion has the effect of enhancing the temperature causing higher flow rates at the surface.

In Figure 3 the concentration profiles decrease as the thermal dispersion coefficient increases for both aiding and opposing buoyancy cases. In the case of a pseudoplastic, the concentration profiles decrease with an increase in the thermal dispersion coefficient 


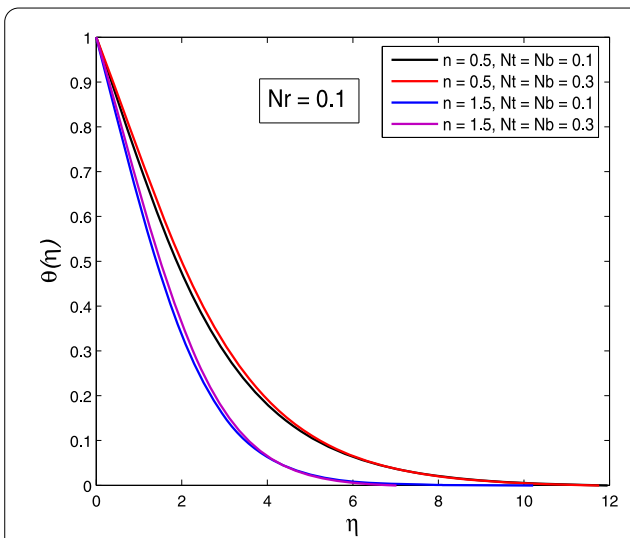

(a)

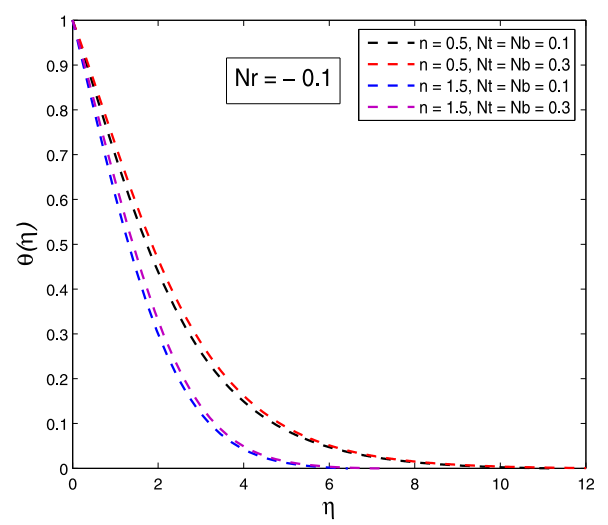

(b)

Figure 5 Variation of temperature profiles with similarity variable $\eta$ for (a) aiding buoyancy $(\mathrm{Nr}>0)$ and (b) opposing buoyancy $(\mathrm{Nr}<0)$, when $\gamma=0.3, \operatorname{Ra}_{d}=1$, Le $=1$.

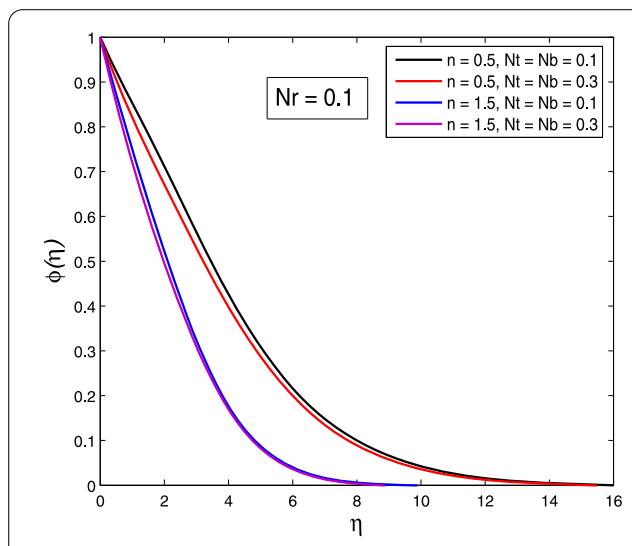

(a)

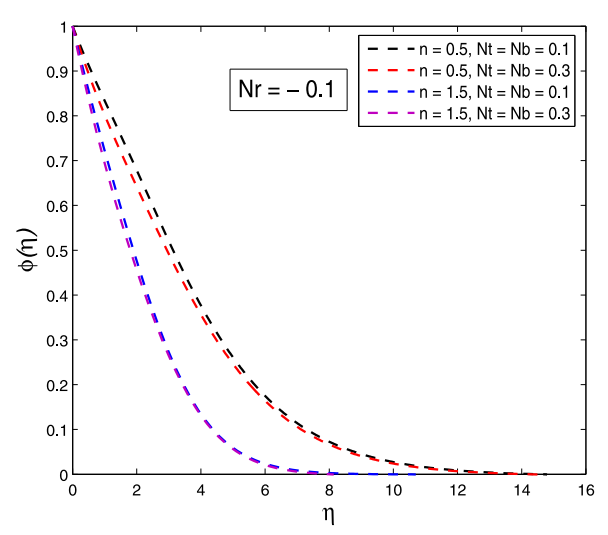

(b)

Figure 6 Variation of concentration profiles with similarity variable $\eta$ for (a) aiding buoyancy $(\mathrm{Nr}>0$ ) and (b) opposing buoyancy $(\mathrm{Nr}<0)$, when $\gamma=0.3, \operatorname{Ra}_{d}=1$, Le $=1$.

more significantly in the case of opposing buoyancy compared to the case of aiding buoyancy.

The variation in velocity, temperature and nanoparticle volume fraction with the powerlaw index, Brownian motion and thermophoresis parameters is shown in Figures 4-6. We observe that both the Brownian motion and the thermophoresis parameters increase the fluid velocity and the temperature for both pseudoplastics and dilatants. The nanoparticle Brownian motion enhances thermal conduction via direct heat transport or microconvection of fluid surrounding individual nanoparticles. For very small solid particles, the Brownian motion is strong. Following earlier studies by Buongiorno [14], Nield and Kuznetsov [5] and others, the thermophoresis parameter values have been selected to simulate realistic applications in heat exchangers, chemical engineering, porous media flows, etc. For particular values of Brownian motion and thermophoresis parameters, an increase in the velocity boundary layer was observed for both aiding and opposing buoyancy cases. The temperature and nanoparticle volume boundary layer thicknesses decrease as the 


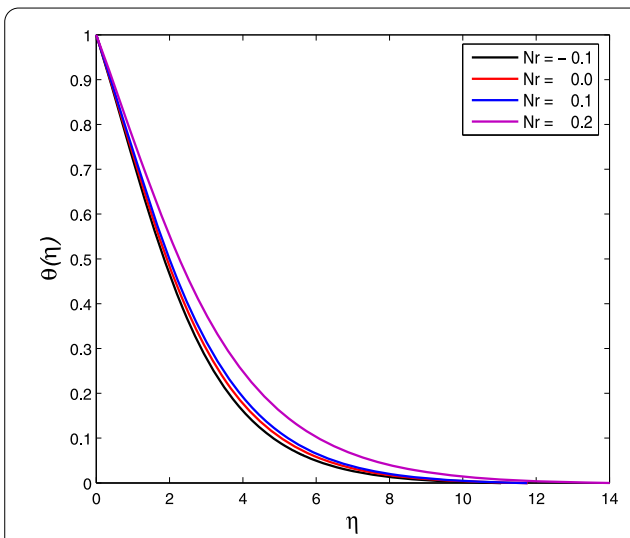

(a)

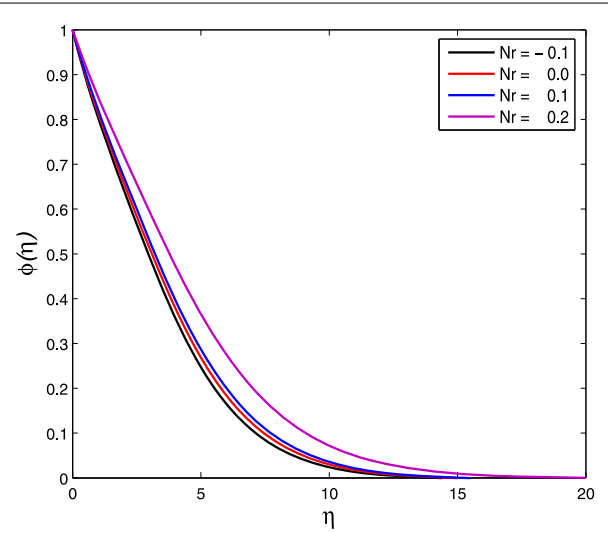

(b)

Figure 7 Variation of (a) temperature and (b) concentration profiles with similarity variable $\eta$ for varying buoyancy (Nr), when $n=0.5, \gamma=0.3, \mathrm{Ra}_{d}=1, \mathrm{Le}=1, \mathrm{Nt}=\mathrm{Nb}=0.3$.

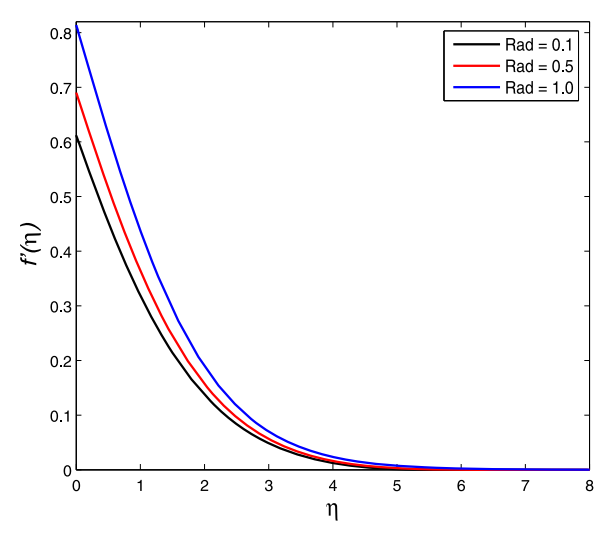

(a)

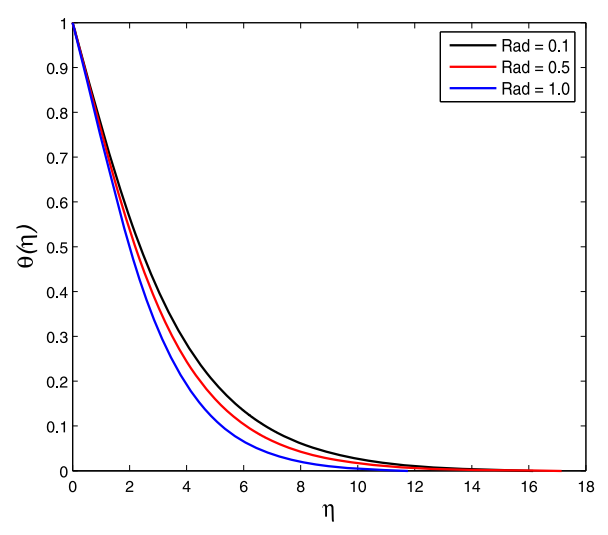

(b)

Figure 8 Variation of (a) velocity and (b) temperature profiles with similarity variable $\eta$ for varying $\mathrm{Ra}_{d}$, when $n=0.5, \gamma=0.3, \mathrm{Nr}=0.1, \mathrm{Le}=1, \mathrm{Nt}=\mathrm{Nb}=0.3$.

power-law index increases. The effect of Brownian motion and thermophoresis parameters on the nanoparticle volume fraction profile is exactly opposite to the temperature profile. Here we observe that the change in nanoparticle volume fraction profiles is less in the case of dilatant fluids compared to pseudoplastics.

The effects of the buoyancy ratio parameter on the temperature and concentration profiles are shown in Figure 7. For $\mathrm{Nr}>0$ the thermal and concentration buoyancy forces act in the same direction and for $\mathrm{Nr}<0$ the thermal buoyancy force acts in the opposite direction to the concentration buoyancy force. The buoyancy effect has a considerable utility in chemical engineering designs of packed bed transport systems. In the case of aiding buoyancy $(\mathrm{Nr}>0)$, an increase in buoyancy has the effect of increasing the induced flow along the surface causing a reduction in the temperature and concentration profiles. The opposite situation is observed in the case of opposing buoyancy flow.

Figures 8 and 9 show the impact of the pore diameter dependent Rayleigh number $\mathrm{Ra}_{d}$ on velocity, temperature and nanoparticle volume fraction when all other parame- 


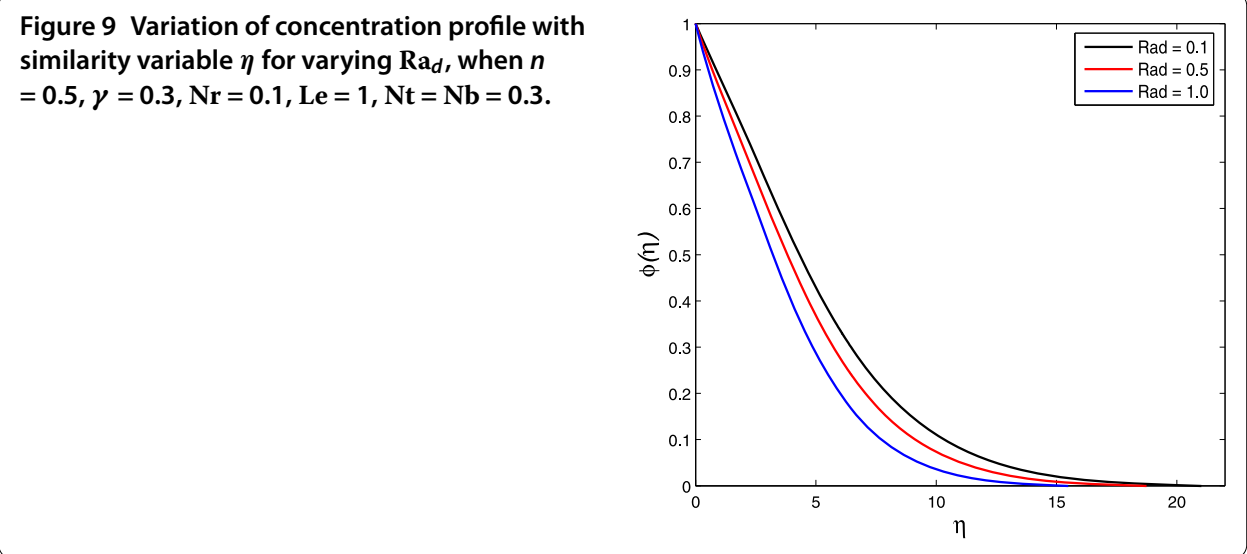

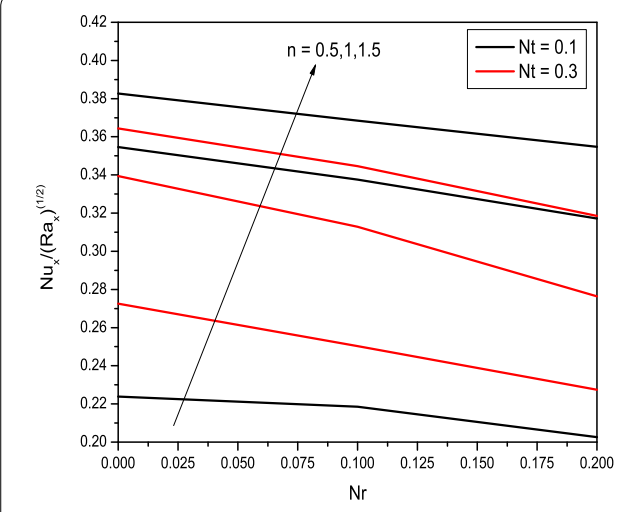

(a)

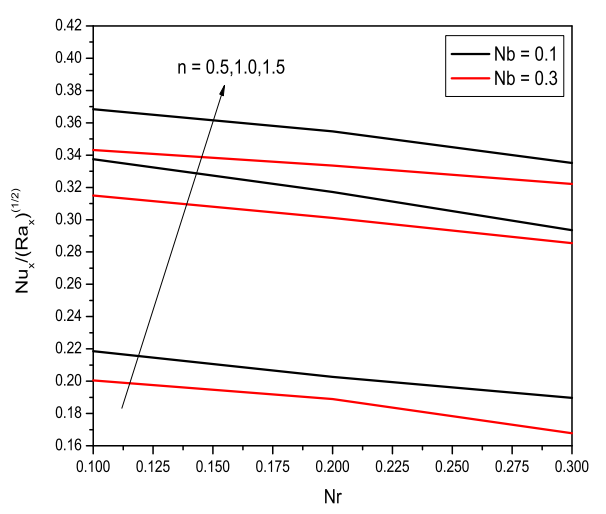

(b)

Figure 10 Variation of the heat transfer coefficient with (a) $\mathrm{Nt}$ and (b) $\mathrm{Nb}$ for varying $\mathrm{Nr}$ for fixed values of $\gamma=0.3, \operatorname{Ra}_{d}=1, \mathrm{Le}=1$.

ters are fixed. The pore diameter dependent Rayleigh number describes the relative intensity of the buoyancy force. In the present study, we have considered values in the range $0.05 \leq \mathrm{Ra}_{d} \leq 1.1$. Figure 8 shows that an increase in $\mathrm{Ra}_{d}$ enhances the velocity boundary layer thickness close to the wall, and has less impact far from that wall. The opposite phenomenon is observed in the case of the thermal and nanoparticle volume fraction boundary layer thicknesses.

The variation of the wall heat transfer coefficient with the buoyancy ratio parameter is shown in Figure 10. It is interesting to note that an increase in the power law index parameter enhances that heat transfer rate. The thermophoresis parameter $\mathrm{Nt}$ values were selected so as to be physically realistic, and are the same as those used by Buongiorno [14]. The values of $\mathrm{Nt}=0.1,0.3$ were also used by Khan and Pop [15]. It can also be noted that for some particular value of power-law index, heat transfer rate decreases with an increase in thermophoresis parameter. Similar type of observation is also found in the case of Brownian motion. The nanoparticle Brownian motion decelerates the flow while promoting heat conduction. Figure 11 shows the effect of $\mathrm{Ra}_{d}$ on the heat transfer rate. The change in the heat transfer rate far from the wall increases when the thermal dispersion decreases. It is interesting to note that an increase in the parameter $\mathrm{Ra}_{d}$ causes a reduction in the heat transfer rate. The effect of $\gamma$ on the heat transfer rate is shown in Figure 11(b). 


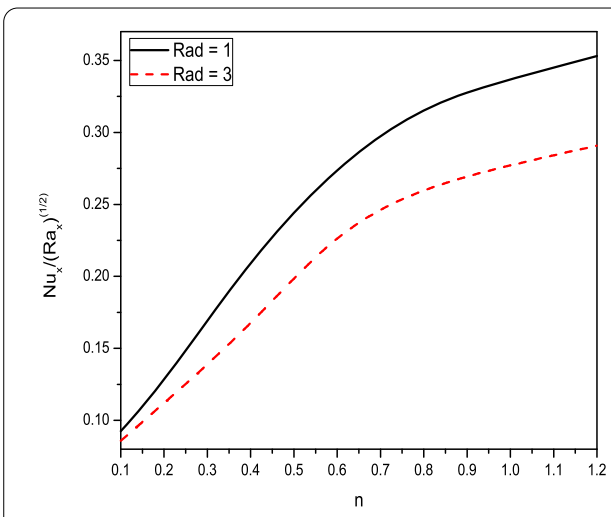

(a)

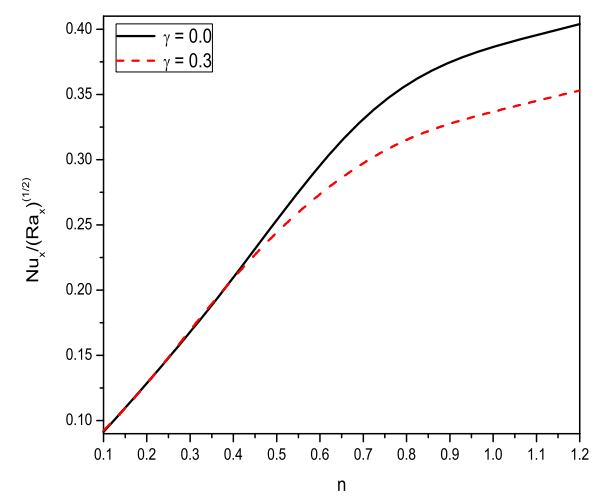

(b)

Figure 11 Variation of the heat transfer coefficient with (a) $\operatorname{Ra}_{d}(\gamma=0.3)$ and (b) $\gamma\left(\operatorname{Ra}_{d}=1\right)$ for varying $n$, with fixed values of $\mathrm{Nr}=0.1, \mathrm{Le}=1, \mathrm{Nt}=\mathrm{Nb}=0.1$.

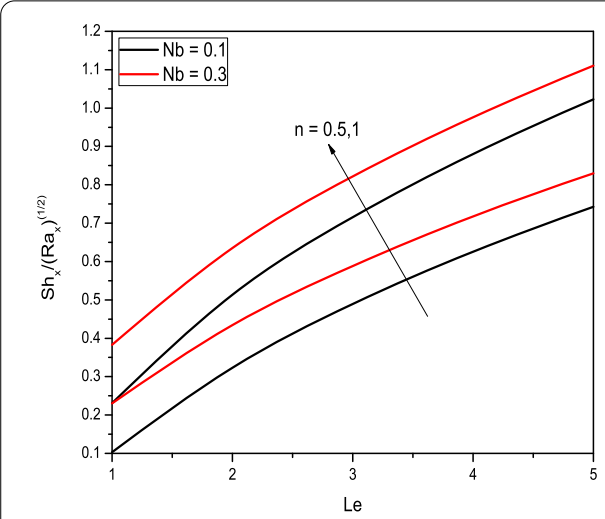

(a)

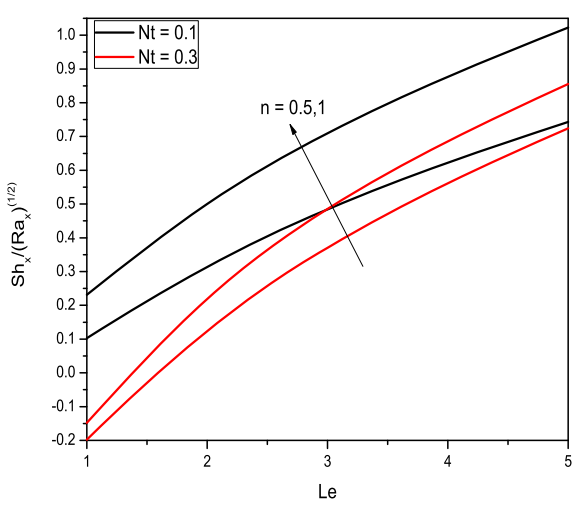

(b)

Figure 12 Variation of the mass transfer coefficient with (a) varying $\mathrm{Nb}$ and (b) varying $\mathrm{Nt}$ for varying values of $\mathrm{Le}$, with fixed values of $\mathrm{Nr}=0.1, \gamma=0.3, \mathrm{Ra}_{d}=1$.

It is clear that up to a certain point, the heat transfer rate increases uniformly regardless of the presence or absence of the thermal dispersion coefficient. Beyond a certain point, the heat transfer rate decreases with an increase in the thermal dispersion coefficient.

The effects of the Lewis number on mass transfer for different values of the power-law index, the Brownian motion and thermophoresis parameters are shown in Figure 12. The Lewis number characterizes fluid flows where there is simultaneous heat and mass transfer due to convection. The effect of an increase in the thermophoresis parameter is to reduce mass transfer. The reverse trend is observed in the case of an increase in the Brownian motion parameter.

\section{Conclusions}

We have presented a study of heat and mass transfer in non-Newtonian nanofluid flow over an impermeable vertical wall in a porous medium. We have further considered the cases of aiding and opposing buoyancy forces. We have presented numerical results for heat and mass transfer rates for different nanoparticle Brownian motion, thermophore- 
sis and power-law index parameters. We have shown, inter alia, that an increase in both Brownian motion and thermophoresis parameters enhances the fluid motion and the temperature for both pseudoplastic and dilatant fluids. An increase in the thermophoresis parameter has the effect of reducing the mass transfer rate.

Competing interests

The authors declare that they have no competing interests.

Authors' contributions

The work including proof reading was done by all the authors.

\section{Acknowledgements}

The authors are grateful to the reviewers for their comments and constructive suggestions. We acknowledge financial support from the University of KwaZulu-Natal.

Received: 10 June 2013 Accepted: 6 September 2013 Published: 18 Nov 2013

\section{References}

1. Shenoy, AV: Non-Newtonian fluid heat transfer in porous media. Adv. Heat Transf. 24, 101-190 (1994)

2. Ellahi, R, Raza, M, Vafai, K: Series solutions of non-Newtonian nanofluids with Reynolds' model and Vogels' model by means of the homotopy analysis method. Math. Comput. Model. 55, 1876-1891 (2012)

3. Rastogi, SK, Poulikakos, D: Double-diffusion from a vertical surface in a porous region saturated with a non-Newtonian fluid. Int. J. Heat Mass Transf. 38, 935-946 (1995)

4. Kairi, RR, Narayana, PAL, Murthy, PVSN: The effect of double dispersion on natural convection heat and mass transfer in a non-Newtonian fluid saturated non-Darcy porous medium. Transp. Porous Media 76, 377-390 (2009)

5. Nield, DA, Kuznetsov, AV: The Cheng-Minkowycz problem for natural convective boundary-layer flow in a porous medium saturated by a nanofluid. Int. J. Heat Mass Transf. 52, 5792-5795 (2009)

6. Uddin, MJ, Yusoff, NHM, Bég, OA, Ismail, Al: Lie group analysis and numerical solutions for non-Newtonian nanofluid flow in a porous medium with internal heat generation. Phys. Scr. 87(1-14), 025401 (2013)

7. Hady, FM, Ibrahim, FS, Abdel-Gaied, SM, Eid, MR: Boundary-layer non-Newtonian flow over vertical plate in porous medium saturated with nanofluid. Appl. Math. Mech. - Engl. Ed. 32, 1577-1586 (2011)

8. Gorla, RSR, Khan, W: Natural convective boundary layer flow over a vertical cylinder embedded in a porous medium saturated with a nanofluid. ASME J. Nanotechnol. Eng. Med. 3(1-5), 034501 (2012)

9. Gorla, RSR, Chamkha, A: Natural convective boundary layer flow over a horizontal plate embedded in a porous medium saturated with a Nanofluid. J. Mod. Phys. 2, 62-71 (2011)

10. Gorla, RSR, Chamkha, A: Natural convective boundary layer flow over a nonisothermal vertical plate embedded in a porous medium saturated with a nanofluid. Nanoscale Microscale Thermophys. Eng. J. 15, 81-94 (2011)

11. Christopher, RH, Middleman, S: Power law flow through a packed tube. Ind. Eng. Chem. Fundam. 4, 422-426 (1965)

12. Dharmadhikari, RV, Kale, DD: Flow of non-Newtonian fluids through porous media. Chem. Eng. Sci. 40, 527-528 (1985)

13. Mohammadien, AA, El-Amin, MF: Thermal dispersion radiation effects on non-Darcy natural convection in a fluid saturated porous medium. Transp. Porous Media 40, 153-163 (2000)

14. Buongiorno, J: Convective transport in nanofluids. ASME J. Heat Transf. 128, 240-250 (2006)

15. Khan, WA, Pop, I: Boundary layer flow of a nanofluid past a stretching sheet. Int. J. Heat Mass Transf. 53, 2477-2483 (2010)

10.1186/1687-2770-2013-243

Cite this article as: Kameswaran and Sibanda: Thermal dispersion effects on convective heat and mass transfer in an Ostwald de Waele nanofluid flow in porous media. Boundary Value Problems 2013, 2013:243

\section{Submit your manuscript to a SpringerOpen ${ }^{\circ}$ journal and benefit from:}

- Convenient online submission

- Rigorous peer review

- Immediate publication on acceptance

- Open access: articles freely available online

- High visibility within the field

- Retaining the copyright to your article 